\title{
Embarazo molar en el Hospital Provincial General Docente Riobamba. Enero 2013-marzo 2018
}

\section{Molar pregnancy in the General Hospital of Riobamba. January 2013-March 2018}

Autoras:

Tatiana Elizabeth Huilca García'

Erica Liseth Lara Sandoval

Anabela del Rosario Criollo Criollo,

'Ministerio de Salud Pública, Riobamba,Ecuador

${ }^{2}$ Universidad Nacional de Chimborazo, Riobamba, Ecuador

${ }^{3}$ Hospital Provincial General Docente de Riobamba, Ecuador

Autora de correspondencia: Erica Liseth Lara Sandoval, Universidad Nacional de Chimborazo, dirección postal: Ave. Antonio José de Sucre Km 1 1⁄2 Vía a Guano, email: ericalls@hotmail.es.

\section{RESUMEN}

La enfermedad trofoblástica gestacional está caracterizada por un crecimiento anómalo de esas células de la placenta luego de una fertilización anormal; lo cual puede presentar alteraciones de tipo premalignas y malignas. Un estudio con enfoque mixto, de tipo observacional descriptivo, transversal permitió alcanzar el propósito de describir la incidencia del embarazo molar en pacientes atendidas en el Hospital Provincial General Docente Riobamba, durante el período comprendido entre enero 2013 y marzo 2018. El universo de estudio estuvo constituido por las historias clínicas de las embarazadas atendidas en el Servicio de Ginecología y Obstetricia, de esa institución de salud en el período mencionado; al que se le practicó un muestreo no probabilístico intencional o deliberado, que permitió seleccionar 53 expedientes de pacientes con diagnóstico certero de embarazo molar. El 66\% de las pacientes en estudio se encuentraban en el rango de 10 a 19.6 semanas al momento de ser diagnosticadas con mola hidatiforme. Existió una alta incidencia de la patología entre las edades de 16 a 25 años con un $64 \%$. La sintomatología más frecuente reportada incluyó sangrado vaginal y dolor abdominal con un total del $(92,45 \%)$. Sin embargo, predominaron las entidades benignas con un $81,13 \%$. El principal factor de riesgo de padecer esta patología resultó el antecedente de abortos previos.

Palabras clave: mola hidatiforme, enfermedad trofoblástica gestacional, gonadotropina coriónica.

\section{ABSTRACT}

Gestational trophoblastic disease is characterized by an abnormal growth of these cells of the placenta after abnormal fertilization; which may present alterations of premalignant and malignant type. A study with a mixed approach, descriptive, cross-sectional observational type, allowed to reach the purpose of describing the incidence of molar pregnancy in patients 
attended in the General Hospital of Riobamba, during the period January 2013- March 2018. The universe of study was constituted by the clinical histories of the pregnant women attended in the Service of Gynecology and Obstetrics of that institution of health mentioned above. 53 records of patients with an accurate diagnosis of molar pregnancy were chosen by an intentional or deliberate non-probabilistic sampling. $66 \%$ of the patients under study were in the range of 10 to 19.6 weeks at the time of being diagnosed with hydatidiform mole. There was a high incidence of pathology between the 16 to 25 years of ages with $64 \%$. The most frequent symptomatology reported included vaginal bleeding and abdominal pain with a total of $92.45 \%$. However, benign entities predominated with $81.13 \%$. The main risk factor of suffering this pathology was the antecedent of previous abortions.

Keywords: Hydatidiform Mole, Gestational Trophoblastic Disease, Chorionic Gonadotropin.

\section{INTRODUCCIÓN}

La enfermedad trofoblástica gestacional (ETG), o mola hidatiforme, se conoce desde tiempos antiguos; Hipócrates en el siglo 4 a.C. describió esta entidad patológica como una hidropesía del útero y se especulaba que era producida por la presencia de aguas corrompidas en ese órgano. De ahí el origen etimológico de la palabra, que deriva del vocablo griego hydatisia, que significa gotas de agua y del vocablo latino mola, que significa falsa concepción. ${ }^{(1-3)}$ Esta patología está caracterizada por un crecimiento anómalo de las células trofoblásticas de la placenta, posteriormente a una fecundación anormal, la cual puede provocar alteraciones de tipo pre maligno y maligno. La mola hidatiforme completa (CHM) y la parcial (PHM) se incluyen en las entidades pre malignas. El estado maligno es conocido como neoplasia trofoblástica gestacional (NTG) y se compone de 4 subtipos. $^{(1)}$

La ETG presenta una incidencia y una epidemiología muy variables, en dependencia de la situación geográfica, la población y el estado socioeconómico; además constituye un problema ginecológico poco frecuente. ${ }^{(2,4)}$

Es alta la incidencia del estado benigno; 1 de cada 1500 embarazos en Europa y Estados Unidos padecen esta complicación, la que resulta notable en América Central, Sudamérica y México, donde se refieren 25 casos por cada 5000 gestaciones. La frecuencia de tipos malignos en Estados Unidos y Europa se presenta en 1 de 20000 a 1 de 40000 embarazos, y entre 1 de 500 a 1 de 1000 embarazos en Asia, África y América Latina. ${ }^{(5)}$

El peligro de sufrir reiterados episodios de mola es del $1.5 \%$ en mujeres con diagnóstico de CHM, pero ante PHM el riesgo aumenta al 2.7 \%. En pacientes menores de 16 años y mayores de 36 años se identifica que es mayor el riesgo de presentar enfermedad trofoblástica gestacional, mientras que en mujeres mayores de 40 años el riesgo se duplica. ${ }^{(6)}$

En la enfermedad trofoblástica gestacional, la edad de las pacientes, los antecedentes de abortos a repetición, la multiparidad, dieta, raza, grupo sanguíneo, inclusive cierta exposición 
a toxinas ambientales como herbicidas, el cigarrillo, el consumo de alcohol y el estado socioeconómico, representan importantes factores de riesgo para desarrollar esta patología. (3) Es amplia la sintomatología de la enfermedad trofoblástica gestacional. Lo más habitual es la presencia de sangrado vaginal ocasional, sin embargo, también se presenta: hiperémesis gravídica, síntomas de hipertiroidismo, trastornos hipertensivos como la preeclampsia, además de alteraciones de las hormonas tiroideas con tendencia al hipertiroidismo, con el valor de T4 elevado y tirotropina u hormona estimulante de la tiroides (TSH) disminuido. No obstante, es raro presenciar tirotoxicosis, pues los niveles de T4 libre posterior a la evacuación del embarazo molar, se normalizan. ${ }^{(6)}$

La ecografía es un parámetro de rutina de control al inicio del embarazo que permite el diagnóstico temprano de ETG, en comparación con la aparición de la sintomatología. ${ }^{(4)}$ Es necesario poder reconocer que en NTG se llega al diagnóstico ante valores anormales de la fracción beta de gonadotropina coriónica humana ( $\beta$-hCG), o a su vez, ante valores bajos que en próximos controles se encuentran exageradamente altos, luego de varias semanas de post evacuación. ${ }^{(7)}$

Para el control, seguimiento y monitoreo de la enfermedad trofoblástica gestacional se utiliza el examen serológico de los niveles de $\beta$-hCG, por lo que es necesario conocer que en ciertos casos se puede originar el síndrome de hCG fantasma. ${ }^{(1)}$

El procedimiento terapéutico para resolver el embarazo molar es la realización de la dilatación y evacuación; ante la posibilidad de perforación del útero se someta a la paciente a legrado por succión. ${ }^{(3)}$

Por el riesgo de desarrollar NTG las pacientes deben ser controladas con la valoración de los niveles de $\beta$-hCG, mientras que para pacientes con diagnóstico confirmado de NTG, el tratamiento que se debe seguir es la quimioterapia. ${ }^{(3,8)}$

Con el objetivo de conocer la extensión de esta patología se impone la realización de un control médico integral, mediante análisis de sangre, con el fin de constatar la función hepática, renal, la biometría hemática y los niveles de $\beta$-hCG. Se propone que se realice un examen físico completo que incluya el examen especular en busca de rasgos de metástasis de tipo vaginal. ${ }^{(1,9)}$

Se debe explicar claramente a la paciente sobre el manejo y procedimientos a los que se va a someter, pues se ha demostrado que el estado psicológico se ve afectado en este proceso; por lo tanto, se recomienda el apoyo de las especialidades de oncología y psiquiatría debido a la naturaleza de la enfermedad..$^{(6,10,11)}$

En el contexto del Hospital Provincial General Docente Riobamba, el estudio de la incidencia del embarazo molar no se encuentra suficientemente descrito; por lo que se decidió realizar un proceso investigativo al respecto, que constituirá una herramienta para la gerencia de esa casa de salud. El valor técnico metodológico aportado facilitará la toma de decisiones administrativas y epidemiológicas en relación con esa patología. 
Atendiendo a lo anterior, las autoras realizaron un estudio con el propósito de describir la incidencia del embarazo molar en pacientes atendidas en el Hospital Provincial General Docente Riobamba, durante el período comprendido entre enero 2013 y marzo 2018.

\section{MATERIAL Y MÉTODOS}

El proceso investigativo se desarrolló en el periodo comprendido entre noviembre 2016 y junio 2017, en el Hospital Provincial General Docente Riobamba; el mismo que pertenece al segundo nivel de atención del Ministerio de Salud Pública, y se ubica en la Av. Félix Proaño y Chile de esta ciudad, en la provincia de Chimborazo-Ecuador. Se realizó a través de un estudio con enfoque mixto, de tipo observacional descriptivo, transversal.

El universo de estudio estuvo constituido por las historias clínicas de las embarazadas atendidas en el Servicio de Ginecología y Obstetricia, de la institución de salud mencionada, durante el período comprendido entre enero 2013 y marzo del 2018. Se realizó un muestreo no probabilístico, de tipo intencional o deliberado que permitió seleccionar 53 historiales de pacientes con diagnóstico de certeza de embarazo molar. Los respectivos documentos rezaban en el departamento de Estadística del Hospital Provincial General Docente Riobamba.

Para este estudio se utilizaron las siguientes variables:

- Lugar de residencia.

- $\quad$ Edad gestacional.

- $\quad$ Edad materna.

- Síntomas más frecuentes.

- Clasificación histopatológica de la mola.

- Abortos previos y multiparidad.

- Anticoncepción.

Se emplearon métodos de nivel teórico y lógico-práctico durante la organización del proceso investigativo, la ejecución de las actividades previstas y la solución de problemas, que incidieron sobre la marcha de la investigación. Además, el método histórico-lógico en el análisis permitió un estudio de manera cronológica en el transcurso del periodo investigado, y el método inductivo-deductivo se utilizó en el estudio para determinar los elementos que intervinieron en el embarazo molar.

A través de la técnica de revisión de documentos se recogieron datos de interés con la ayuda de un formato prediseñado. Para la realización del proceso investigativo en el contexto de investigación, se solicitó la debida autorización a las correspondientes instancias del Hospital General Docente de la ciudad de Riobamba.

Los datos recolectados fueron organizados en una base de datos elaborada en el programa Microsoft Excel, el cual facilitó el procesamiento y la interpretación utilizando técnicas de estadística descriptiva. Los resultados se presentaron utilizando tablas estadísticas que permitieron la debida interpretación. 
Las investigadoras tuvieron en cuenta los principios bioéticos establecidos por las convenciones internacionales: beneficencia, autonomía, justicia y no maleficencia.

\section{RESULTADOS}

El análisis de las variables estudiadas respecto a la incidencia de embarazo molar permitió realizar la caracterización de esa entidad en el contexto de investigación.

Tabla 1. Lugar de residencia

\begin{tabular}{|l|r|r|}
\hline Lugar de residencia & \multicolumn{1}{|c|}{ No. } & \multicolumn{1}{c|}{ \% } \\
\hline Alausí & 3 & 5.66 \\
\hline Cacha & 1 & 1.89 \\
\hline Cajabamba & 1 & 1.89 \\
\hline Calpi & 1 & 1.89 \\
\hline Cebadas & 1 & 1.89 \\
\hline Chambo & 5 & 9.43 \\
\hline Colta & 2 & 3.77 \\
\hline Guamote & 7 & 13.21 \\
\hline Guano & 2 & 3.77 \\
\hline Licto & 1 & 1.89 \\
\hline Pallatanga & 5 & 9.43 \\
\hline Palmira & 1 & 1.89 \\
\hline Píllaro & 1 & 1.89 \\
\hline Riobamba & 20 & 37.74 \\
\hline Tambo & 1 & 1.89 \\
\hline Yaruquíes & 1 & 1.89 \\
\hline TOTAL & 53 & 100.00 \\
\hline
\end{tabular}

De las pacientes en estudio, 20 de aquellas proceden de la cabecera cantonal de la provincia, para un 38\%. Es destacable que a pesar de ser Guamote un cantón pequeño en comparación con los diferentes lugares de procedencia de las pacientes en estudio, representó el 13.21\% correspondiente a 7 pacientes (tabla 1).

Tabla 2. Edad gestacional

\begin{tabular}{|c|r|c|}
\hline $\begin{array}{c}\text { Edad gestacional } \\
\text { (semanas) }\end{array}$ & No. & \% \\
\hline $1-9,6$ & 13 & 24.53 \\
\hline $10-19,6$ & 35 & 66.04 \\
\hline $20-29,6$ & 3 & 65.66 \\
\hline$>30$ & 2 & 43.77 \\
\hline TOTAL & 53 & 100.00 \\
\hline
\end{tabular}


El 66\% de las pacientes en estudio se encontraron entre el rango de 10 a 19.6 semanas al momento de ser diagnosticadas con mola hidatiforme, mientras que las pacientes con 30 semanas fueron las menos representadas, con un 4\% (tabla 2).

Gráfico 1. Edad materna en años cumplidos

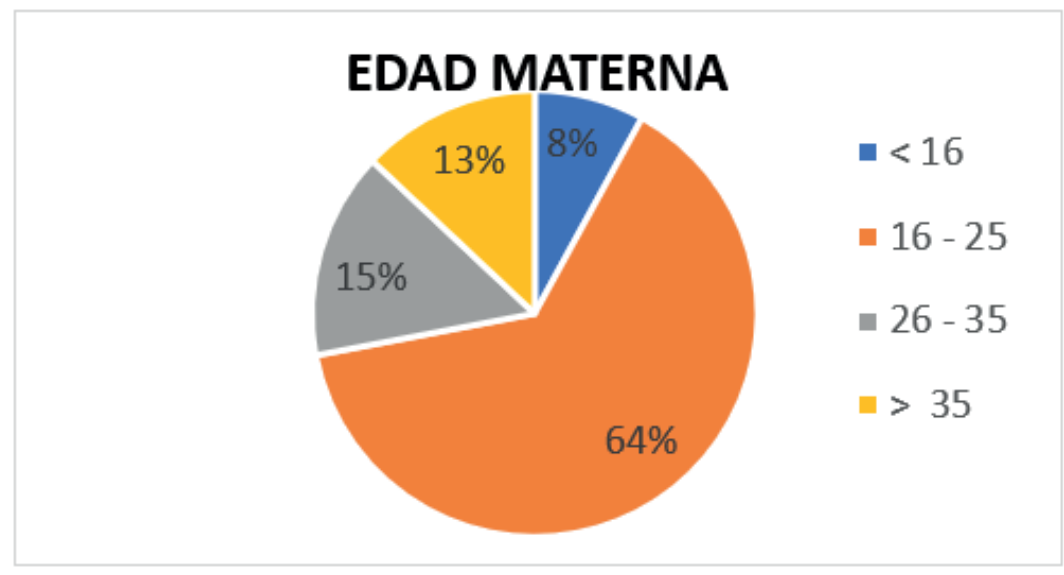

En lo referente a la edad materna se puede identificar que 34 pacientes representadas en un $64 \%$ están comprendidas entre las edades de 16 a 25 años, lo que refleja una alta incidencia en este rango (gráfico 1).

Dentro de la población en estudio, la sintomatología más frecuente que se observó fue el sangrado vaginal y el dolor abdominal $(92,45 \%)$.

Tabla 3. Características histopatológicas

\begin{tabular}{|c|r|r|r|r|r|}
\hline \multicolumn{7}{|c|}{ Histopatológico } \\
\hline Año & Benigno & Maligno & Sin reporte & Otros & Total \\
\hline 2013 & 5 & - & 3 & 1 & 9 \\
\hline 2014 & 13 & - & 2 & - & 15 \\
\hline 2015 & 8 & 1 & - & - & 9 \\
\hline 2016 & 10 & 1 & 1 & - & 12 \\
\hline 2017 & 7 & - & - & - & 7 \\
\hline 2018 & - & - & 1 & - & 1 \\
\hline TOTAL & 43 & 2 & 7 & 1 & 53 \\
\hline
\end{tabular}

En relación con los resultados histopatológicos, predominaron las entidades benignas para un $81,13 \%$, mientras que el tipo maligno solamente se evidenció en dos casos. La mayor incidencia se reportó en el año 2014; sin embargo, en este no se diagnosticó ninguna de las entidades malignas (tabla 3 ). 
Tabla 4. Abortos previos y multiparidad

\begin{tabular}{|l|r|r|}
\hline \multicolumn{1}{c|}{$\begin{array}{c}\text { Características } \\
\text { ginecoobstétricas }(\mathbf{n}=\mathbf{5 3})\end{array}$} & $\mathbf{N}^{\mathbf{0}}$ & $\mathbf{\%}$ \\
\hline ABORTOS & 11 & 20,75 \\
\hline GESTAS & 28 & 52,83 \\
\hline NINGUNA & 25 & 47,17 \\
\hline PRIMIGESTA & 10 & 18,88 \\
\hline BIGESTA & 8 & 15,09 \\
\hline TRIGESTA & 2 & 3,77 \\
\hline MULTIGESTA & 8 & 15,09 \\
\hline
\end{tabular}

Únicamente 11 pacientes $(20,75 \%)$ de la población en estudio presentaron antecedentes de abortos previos y, además, se señala que las primigestas mostraron un rango elevado de 10 pacientes $(18,88 \%)$, lo que nos permite deducir que no influye directamente en la predisposición de esta patología (tabla 4).

Gráfico 2. Anticonceptivos

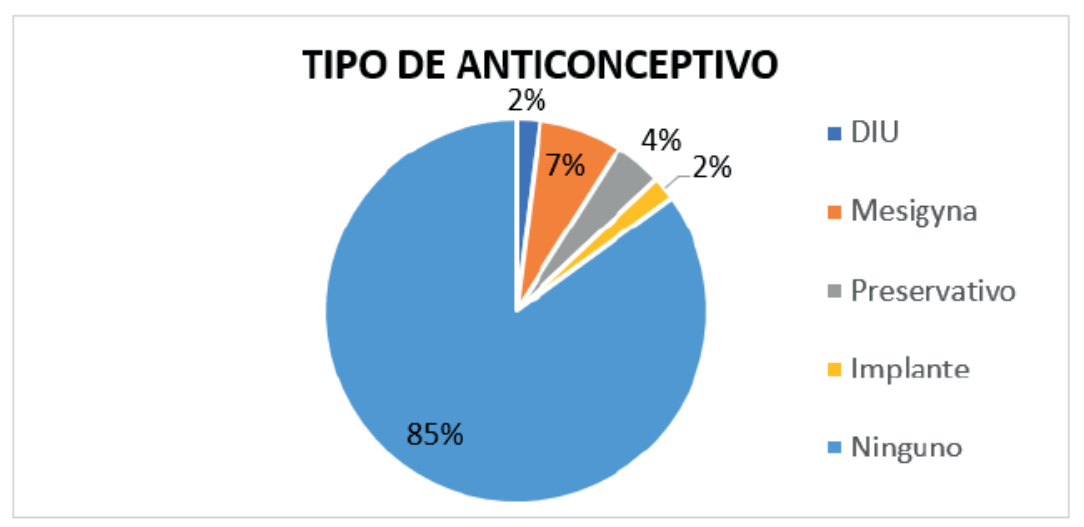

Dentro de la investigación se demostró que 45 pacientes representadas en el $85 \%$ de la población en estudio, no utilizaron ningún método anticonceptivo que haya favorecido la aparición de esta entidad patología (gráfico 2).

\section{DISCUSIÓN}

El predominio de pacientes que presentan esta entidad procede del cantón Riobamba (cabecera provincial). Al respecto, algunos autores ${ }^{(4)}$ destacan la importancia de la ubicación geográfica en relación con la atención de salud, al considerar determinados parámetros que 
pueden contribuir con la etiología de las diferentes enfermedades. Mientras que desde otro punto de vista, Calles ${ }^{(12)}$ habla sobre el predominio en la zona urbana difiriendo con el estudio que se presenta.

La edad materna representa un factor de riesgo de relevancia en relación con la mola hidatidiforme. En el estudio que se presenta, el $64 \%$ de las pacientes ingresadas con ese diagnóstico de embarazo tenían edades de 16 a 25 años; lo que se corresponde con los resultados observados en el estudio titulado "Incidental Finding of Persistent Hydatidiform Mole in an Adolescent on Depo-Provera". ${ }^{(13)}$ En esa investigación, la mayoría de embarazos molares acontecieron en edades reproductivas de 19 a 34 años. Además, refiere que, en adolescentes $(<20$ años), la probabilidad es siete veces mayor, siendo el doble para las mayores que 40 años. ${ }^{(13)}$ La sintomatología más frecuente fue sangrado vaginal y dolor abdominal representando el $92.45 \%$, en relación a 4 mujeres con esta patología que acudieron con clínica diferente. Estos datos guardan relación con el artículo "Clinico-epidemiological profile of molar pregnancies in a tertiary care centre of Eastern Nepal: a retrospective review of medical récords" ${ }^{(14)}$ donde sobresalió la presencia anormal de sangrado uterino como el síntoma más usual (86.3\%); de igual manera, coinciden con los datos publicados por Enrique et al., ${ }^{(15)}$ quien obtuvo el 97\% de pacientes que ingresaban con este síndrome.

En el reporte histopatológico se indica que las entidades benignas se presentaron en el $81.13 \%$ de pacientes, lo cual guarda relación con el artículo "Caracterización de la enfermedad trofoblástica gestacional en Camagüey"(5) refiriendo que la frecuencia de las formas benignas es alta, mientras que las malignas ocurren con una frecuencia de 1 en 20000 a 1 en 40000 embarazos en Estados Unidos y Europa, la misma que se asemeja a valores encontrados en el estudios de Coriocarcinoma postgestacional, el cual indica que en Europa y Norteamérica se estima 3/100000, aunque en Asia esas estadísticas se elevan de 5-200/100000 ${ }^{(16)} \mathrm{Al}$ respecto, los hallazgos del estudio que se presenta permitieron observar un predominio de la mola hidatiforme completa y una ausencia de la parcial, la cual no concuerda con la Dra. Elisa Calles ${ }^{(12)}$ que reporta en su estudio el $9 \%$ de casos.

Solamente, 11 pacientes $(20,75 \%)$ presentaron antecedentes de abortos previos, lo que reveló que no influye directamente con la aparición de dicha patología. Esto concuerda con Calles $^{(12)}$ y su estudio en el Salvador que revela un bajo porcentaje de pacientes con este antecedente. La paridad no es un factor de riesgo para presentar esta patología ya que en el estudio se pudo apreciar el 18,88\% en las primigestas y en menor porcentaje las multigestas.

El 66\% de las pacientes en estudio se encuentran entre el rango de 10 a 19.6 semanas de gestación, cuando han sido diagnosticadas de la patología, mientras que solo el 4\% llega a sobrepasar las 30 semanas de embarazo. Esa información coincide con la publicada por Sociedad Española de Oncología Médica en su Guía de Práctica Clínica para la Atención a la Enfermedad Trofoblástica Gestacional de 2017, ${ }^{(17)}$ en la que se plantea que la ecografía de rutina al inicio del embarazo es un parámetro de suma importancia en el control del mismo, mediante la cual se puede identificar y diagnosticar esta entidad a tiempo para la posterior toma de acciones. Lo anterior también concuerda con el artículo titulado Caracterización de 
la enfermedad trofoblástica gestacional en Camagüey ${ }^{(5)}$ en el que se reporta que el $65,4 \%$ de pacientes diagnosticadas antes de las 12 semanas.

\section{CONCLUSIONES}

- La ETG en el Hospital Provincial General Docente de Riobamba durante el periodo de enero 2013 a marzo de 2018 predominó en pacientes con edades comprendidas entre 16 y 25 años. Los síntomas más comunes fueron el sangrado vaginal y el dolor abdominal.

- El principal factor de riesgo de padecer esta patología resultó el antecedente de abortos previos.

- Con respecto a las complicaciones de las pacientes, se evidenció insuficientes controles y seguimientos.

Conflictos de intereses: las autoras declaran que no existen.

Declaración de contribuciones: todos los autores recolectaron, analizaron e interpretaron los datos, además de confeccionar el artículo científico.

\section{REFERENCIAS BIBLIOGRÁFICAS}

1. Santaballa A, García Y, Fuentes J, Herrero A, Laínez N, De Juan A, et al. SEOM clinical guidelines in gestational trophoblastic disease. Clinical and Translational Oncology [Internet]. 2017 [citado: 2018 Mayo 02]; 20(1): 38-46. Disponible en: https://www.ncbi.nlm.nih.gov/pmc/articles/PMC5785593/.

2. Cabero Roura L, Saldivar Rodriguez D. Operatoria Obstétrica.Una visión actual [Internet]. Primera ed. México: Médica Panamericana; 2009 [citado: 2018 Mayo 03]. Disponible en: https://books.google.com.ec/books? id=n 7 qU 5 f6 W 9 q IC\&printsec=frontcover $\& \mathrm{hl}=\mathrm{es} \# \mathrm{v}=$ onepage $\& \mathrm{q} \& \mathrm{f}=$ false.

3. Durón González R, Bolaños Morera P. Enfermedad trofoblástica gestacional. Medicina Legal de Costa Rica [Internet]. 2018 [citado: 2018 Mayo 05]; 35(1): 30-43. Disponible en: ht t p : / / w w w.scielo.sa.cr/scielo.php?script=sci_arttext\&pi$\mathrm{d}=\mathrm{S} 1409-00152018000100030 \& \operatorname{lng}=\mathrm{en} \& \mathrm{t} \operatorname{lng}=\mathrm{pt}$.

4. Stevens FT, Katzorke N, Tempfer C, Kreimer U, Bizjak GI, Fleisch MC \& Fehm TN. Gestational Trophoblastic Disorders: An Update in 2015. Geburtshilfe Und Frauenheilkunde [Internet]. 2015 [citado: 2018 Mayo 07]; 75(10): 1043-50. Disponible en: doi:http://doi.or$\mathrm{g} / 10.1055 / \mathrm{s}-0035-1558054$.

5. Cabrera Figueredo I, Valdivieso Y, Fonseca D, Amador de Varona D, Rodriguez D. Caracterización de la enfermedad trofoblástica gestacional en Camagüey [Internet]. 2014 [citado: 2018 Mayo 09]; 54(257): 25-35. Disponible en: http://www.rev16deabril.sld.cu/index.php/16_04/article/view/51. 
6. Espinoza Cárdenas D, Beltrán Vidal L, Pozo Ocampo D. Caso Clínico: Mola Hidatiforme Parcial.Resolución Obstétrica. Revista Médica HJCA [Internet]. 2017 [citado 2018 Mayo 12]; 9(3): 291-295. Disponible en: doi:http://dx.doi.org/10.14410/2017.9.3.cc.47.

7. Fagnoni Bollo JC. Diagnóstico y tratamiento: Enfermedad trofoblástica gestacional. Arch GyO [Internet]. 2015 [citado: 2018 Mayo 16]; 53(3): 36-50. Disponible en: https://www.sguruguay.org/img/noticias/AGO\%2053_3\%20web.pdf.

8. Hernández-Flores SE, Vega-Memije ME, Niebla-Cárdenas D, Audifred Salomón JR, Hal Ramírez WB. Incidencia de enfermedad trofoblástica gestacional. Ginec Obst Mex [Internet]. 2016 [citado 2018 Mayo 20]; 84(6): 377-382. Disponible en: http://www.medigraphic.com/pdfs/ginobsmex/gom-2016/gom166g.pdf.

9. Arriagada DR, Novoa RR. Mola hidatidiforme completa con preeclampsia e hipertiroidismo: presentación clásica. Rev Chil OyG [Internet]. 2017 [citado 2018 Mayo 25]; 82(1): 77-79. Disponible en: doi:http://dx.doi.org/10.4067/S0717-75262017000100011.

10. Colón J, De Ponte A, Serrano R, Aponte A. Mola hidatiforme recurrente asociada a mutación del gen NLRP7: a propósito de un caso. Rev OyG Ven [Internet]. 2015 [citado: 2018 Junio 05]; 75(4): 280-283. Disponible en: http://www.scielo.org.ve/scielo.php?scrip$\mathrm{t}=\mathrm{sci}$ _arttext\&pid=S0048-77322015000400008\&lng=es\&tlng=es.

11. Bruce S, Sorosky J. Gestational Trophoblastic Disease. StatPearls [Internet]. 2017; [citado: 2018 Junio 02]. Disponible en: https://www.ncbi.nlm.nih.gov/books/NBK470267/.

12. Calles-Ramírez E. Perfil epidemiológico, evolución clínica y complicaciones en pacientes con enfermedad gestacional del trofoblasto en el hospital nacional especializado de maternidad enero- diciembre 2013 [tesis de grado]. [San Salvador]: Universidad del Salvador; 2015.

13. Akinlaja O, McKendrick R, Mashak Z, Nokkaew M. Incidental Finding of Persistent Hydatidiform Mole in an Adolescent on Depo-Provera [Internet]. Epub 2016 Dic [citado 2018 Jun 05]. Disponible en: https://www.hindawi.com/journals/criog/2016/6075049/. http://dx.doi.org/10.1155/2016/6075049.

14. Agrawal N, Sagtani RA, Budhathoki SS, and Pokharel HP. Clinico-epidemiological profile of molar pregnancies in a tertiary care centre of Eastern Nepal: a retrospective review of medical récords. Gynecol Oncol Res Pract [Internet]. 2015 [citado 16 Dic 2017]; 2: 9. Disponible en: https://www.ncbi.nlm.nih.gov/pmc/articles/PMC4881004/. DOI: 10.1186/s40661-015-0017-y.

15 Vázquez Y, Brito A, Delgado L, Vázquez E. Caracterización clínica de la enfermedad trofoblástica gestacional en el Hospital ginecobstetrico Ramón Gonzales Coro. Rev Cubana Hig Epidemiol [Internet]. 2014 [citado 2018 Abr 13]; 52(2). Disponible en: http://scielo.sld.cu/scielo.php?script=sci_arttext\&pid=S1561-30032014000200004).

16 Agustín A, Savirón R, Lerma D, Herrero A, Campillos JM. Coriocarcinoma postgestacional. Rev. chil. obstet. ginecol [Internet]. 2015 Ago [citado 2018 Jul 05]; 80(5): 405-411. Disponible en: 
https://scielo.conicyt.cl/scielo.php?script=sci_arttext\&pid=S0717-75262015000500009\&lng=es. http://dx.doi.org/10.4067/S0717-75262015000500009.

17 Santaballa A, García Y, Herrero A, Laínez N, Fuentes J, De Juan A, et al. SEOM clinical guidelines in gestational trophoblastic disease Published online 2017. Clin Transl Oncol [Internet]. 2018 [citado 23 May 2018]; 20(1): 38-46. Disponible en: https://www.ncbi.nlm.nih.gov/pmc/articles/PMC5785593/. DOI:10.1007/s12094-017-1793-0.

Recibido: 13 de julio de 2018

Aceptado: 17 de agosto de 2018 